\title{
Engineering the magnetic coupling and anisotropy at the molecule-magnetic surface interface in molecular spintronic devices
}

Victoria E. Campbell', Monica Tonelli ${ }^{1}$, Irene Cimatti ${ }^{1}$, Jean-Baptiste Moussy², Ludovic Tortech ${ }^{3}$, Yannick J. Dappe ${ }^{2}$, Eric Rivière ${ }^{1}$, Régis Guillot ${ }^{1}$, Sophie Delprat ${ }^{4}$, Richard Mattana ${ }^{4}$, Pierre Seneor ${ }^{4}$, Philippe Ohresser ${ }^{5}$, Fadi Choueikani ${ }^{5}$, Edwige Otero ${ }^{5}$, Florian Koprowiak ${ }^{6}$, Vijay Gopal Chilkuri ${ }^{6}$, Nicolas Suaud ${ }^{6}$, Nathalie Guihéry ${ }^{6}$, Anouk Galtayries ${ }^{7}$, Frederic Miserque ${ }^{8}$, Marie-Anne Arrio ${ }^{9}$, Philippe Sainctavit ${ }^{5,9}$ \& Talal Mallah ${ }^{1}$

\begin{abstract}
A challenge in molecular spintronics is to control the magnetic coupling between magnetic molecules and magnetic electrodes to build efficient devices. Here we show that the nature of the magnetic ion of anchored metal complexes highly impacts the exchange coupling of the molecules with magnetic substrates. Surface anchoring alters the magnetic anisotropy of the cobalt(II)-containing complex (Co(Pyipa) $)_{2}$, and results in blocking of its magnetization due to the presence of a magnetic hysteresis loop. In contrast, no hysteresis loop is observed in the isostructural nickel(II)-containing complex (Ni(Pyipa) $)_{2}$ ). Through XMCD experiments and theoretical calculations we find that $\mathrm{Co}(\text { Pyipa })_{2}$ is strongly ferromagnetically coupled to the surface, while Ni(Pyipa) ${ }_{2}$ is either not coupled or weakly antiferromagnetically coupled to the substrate. These results highlight the importance of the synergistic effect that the electronic structure of a metal ion and the organic ligands has on the exchange interaction and anisotropy occurring at the molecule-electrode interface.
\end{abstract}

\footnotetext{
${ }^{1}$ Institut de Chimie Moléculaire et des Matériaux d'Orsay (ICMMO), CNRS, Université Paris Sud, Université Paris Saclay, 91405 Orsay, France. ${ }^{2}$ SPEC, CEA CNRS, Univesité Paris Saclay, CEA Saclay, 91191 Gif-sur-Yvette, France. ${ }^{3}$ IPCM, UMR CNRS 7201, UPMC, Université Pierre et Marie Curie, F-75005 Paris, France. ${ }^{4}$ Unité Mixte de Physique CNRS/Thales, 1 Avenue Auguste Fresnel, 91767 Palaiseau, France and Université Paris-Sud, 91405 Orsay, France.

${ }^{5}$ Synchrotron SOLEIL, L'Orme des Merisiers Saint-Aubin-BP 48, 91192 Gif-sur-Yvette, France. ${ }^{6}$ Laboratoire de Chimie et Physique Quantiques, Université de Toulouse III, 118, route de Narbonne, 31062 Toulouse, France. ${ }^{7}$ PSL Research University, Chimie ParisTech-CNRS, Institut de Recherche de Chimie Paris, F-75005 Paris, France. ${ }^{8}$ CEA/DEN/DANS/DPC/SCCME, Laboratoire d'Etude de la Corrosion Aqueuse, F-91191 Gif-sur-Yvette, France. ${ }^{9}$ IMPMC-CNRS, Université Pierre et Marie Curie, F-75005 Paris, France. Correspondence and requests for materials should be addressed to V.E.C.

(email: victoria.campbell@u-psud.fr) or to T.M. (email: talal.mallah@u-psud.fr).
} 
W ith the quest to design electronic devices ever smaller, molecules have gained a place in the realm of not only traditional electronics, but also in spintronics. Molecular spintronics is a multidisciplinary field of research that unites the exceptional properties of molecules with the requirements of spin-based technology ${ }^{1,2}$. It has been shown that the magnetoresistance response of a molecular spintronic device hinges upon the interface between the ferromagnetic surface and the molecular layer ${ }^{3}$. Mastering the quality and the nature of this interface is of paramount importance for the construction of devices with reproducible responses ${ }^{3}$. A major challenge is to build devices with defined chemical bonding between the magnetic molecules and the magnetic electrodes allowing a control over the nature of the exchange coupling interaction and thus over the magnetoresistance response of the device ${ }^{4}$. Single magnetic molecules with designed geometry, architecture and electronic structure allow scientist to attain this goal ${ }^{5,6}$.

Recent endeavours towards molecular spintronics include (but are not limited to) the following examples. Mannini and co-workers demonstrated that the magnetic memory effect is retained at low temperature $(<1.0 \mathrm{~K})$ when single molecule magnets (SMMs) are wired not only onto non-magnetic gold surfaces ${ }^{7,8}$ but also onto magnetic surfaces commonly used as ferromagnetic electrodes in spintronic devices, such as the oxide lanthanum strontium manganite (LSMO) and metallic cobalt ${ }^{9}$. When ferromagnetic surfaces were employed, terbium(III) double-decker $\left(\mathrm{TbPc}_{2}\right)$ molecules organized with different orientations (parallel or perpendicular) depending on the surface employed and did not show significant magnetic interaction between the molecules and the substrate ${ }^{9}$. In alternative study, Rizzini et al. ${ }^{10}$ demonstrated that it is possible to induce exchange bias in a small fraction of the $\mathrm{TbPc}_{2}$ molecules adsorbed onto a manganese surface. Another class of materials, paramagnetic porphyrin molecules (Mn-porphyrin and $\mathrm{Fe}$ porphyrin) and phthalocyanine, are known to physically adsorb onto ferromagnetic $\mathrm{Ni}$ or Co surfaces and show surface induced magnetic ordering and hysteresis ${ }^{5,11-16}$.

Although these systems are promising, interface engineering is necessary to fully control and comprehend the nature of the interaction between the magnetic molecular layer and the ferromagnetic surface. To do so, we design metal-containing $(\mathrm{M}=\mathrm{Ni}(\mathrm{II})$ and $\mathrm{Co}(\mathrm{II}))$ magnetic complexes that can chemically bond to epitaxial $\mathrm{Fe}_{3} \mathrm{O}_{4}$ (111), which is chosen as the magnetic substrate, thus defining the interface between the molecules and the electrodes. This step allows us performing the analysis of the exchange coupling interaction responsible for the magnetoresistance response. Phosphonates $\left(\mathrm{R}-\mathrm{PO}(\mathrm{OH})_{2}\right)$ are known to react with a variety of metal-oxides and form stable monolayers through a heterocondensation reaction that results in strong $\mathrm{Fe}-\mathrm{O}-\mathrm{P}(\mathrm{O}(\mathrm{OH}))-\mathrm{R}$ bonds and the release of water molecules ${ }^{17}$. Taking into account these design criteria, we prepare, on one hand, metal complexes bearing phosphonate groups able to make coordination bonds with a metal-oxide surface, and on the other hand, we choose the $\mathrm{Fe}_{3} \mathrm{O}_{4}$ (111) epitaxial metal oxide surface as the magnetic substrate in order not only to ensure stable coordination bond with the designed complex but also to privilege as much as possible its orientation. Surface anchoring induces, in the paramagnetic cobalt(II)-containing complex, a magnetic hysteresis at the Co-edge evidenced by X-ray magnetic circular dichroism (XMCD) studies and indicative of magnetic coupling with the magnetic surface. We do not observe hysteresis at the Ni-edge for the isostructural nickel(II)-containing complex consistent with the absence of magnetic interaction with the surface. First principle $a b$ initio calculations indicate that the $\mathrm{Co}(\mathrm{II})$ complex is relatively strongly ferromagnetically coupled with the surface, while the $\mathrm{Ni}(\mathrm{II})$ one undergoes a much weaker exchange coupling that is antiferromagnetic. These results highlight the fundamental effect that the electronic structure of a metal ion in conjunction with the nature of the organic spacer has on the resulting molecule/magnetic surface interaction.

\section{Results}

Design and synthesis of the molecules. When designing a versatile system that could be exploited in molecular spintronic applications such as spin filtering or spin transfer torque, we took into consideration two main factors. First, we wanted to construct a well-defined molecules/substrate interface by using anchoring groups as spacers that would graft reproducibly and in a controlled manner onto the oxide of choice. This feature, in principle, will allow engineering the molecule/magnetic surface magnetic and electronic interactions. Second, these complexes must be air stable and more importantly thermodynamically stable in solution to prevent possible reactions between the metal ion, belonging to the complex, and the surface.

Many different anchoring groups are known to graft to oxide surfaces such as phosphonates, carboxylates and methyl silanes $^{18,19}$. Amongst them all, the phosphonic acid moiety was chosen because it is known to covalently tether to a variety of oxides (for example $\mathrm{TiO}_{2}, \mathrm{Ta}_{2} \mathrm{O}_{5}$, LSMO, $\mathrm{ZnO}$ and $\left.\mathrm{Fe}_{3} \mathrm{O}_{4}\right)^{20-22}$, is stable in a variety of solvents, in a large range of $\mathrm{pH}$, and is insensitive to hydrolysis ${ }^{17,23,24}$

The organic ligand and the related complexes were designed with three criteria in mind: (i) maximum thermodynamic stability, (ii) facile modification of the nature of the magnetic ion keeping everything else unchanged, and (iii) optimal orientation of the phosphonic acid groups that play the role of spacer units between the magnetic ions and the substrate. This last criterion enables the electronic communication between the surface and the magnetic complexes, and directly impacts the nature and the magnitude of their exchange coupling. The one pot reaction between 2-(aminoethyl)phosphonic acid (2 equiv.), 2-pyridinecarboxaldehyde (2 equiv.) and the corresponding hydrated $\mathrm{M}^{\mathrm{II}}\left(\mathrm{CH}_{3} \mathrm{COO}\right)_{2}$ salt (1 equiv.), using the metal ion $\mathrm{M}^{\mathrm{II}}\left(\mathrm{Co}^{\mathrm{II}}\right.$ or $\left.\mathrm{Ni}^{\mathrm{II}}\right)$ as a template, produced bisimine complexes Coand $\mathrm{Ni}$ (Pyipa) $)_{2}$ as the uniquely observed products (Fig. 1, the tridentate ligand Pyridin-2-ylmetylene)amino]ethyl phosphonic acid (Pyipa) is produced in situ, for full characterization of the complexes see Methods, and Supplementary Discussion).

It is important to notice (Fig. 1) that the organic ligand Pyipa was designed to be tridendate ensuring a large thermodynamic (entropic) stability of the $\mathrm{M}$ (Pyipa) $)_{2}$ complexes. Furthermore, the assembly of two Pyipa ligands by the metal ions leads to only one possible isomer with the phosphonate groups pointing in the same direction (Fig. 1). The two $\mathrm{OH}$ species belonging to the two phosphonate groups are expected to play the role of a 'chelate' towards the $\mathrm{Fe}_{3} \mathrm{O}_{4}$ (111) epitaxial surface, thus bringing an additional (entropic) thermodynamic stabilization to the formation of the complexes/substrate coordination bonds. And finally, the bulkiness of the complex is expected to preclude its large tilting when anchored to the surface, keeping its $\mathrm{C}_{2}$ symmetry axis very close to perpendicular to the substrate.

Single crystal X-ray analysis revealed that $\mathrm{Co}-$ and $\mathrm{Ni}(\text { Pyipa })_{2}$ are isostructural (Fig. 2; Supplementary Table 1; Supplementary Data 1-2). In both complexes the metal centre is bound to the two tridentate ligands, leading to a distorted octahedral environment and molecular $\mathrm{C}_{2 \mathrm{v}}$ symmetry. One oxygen atom of each phosphonate group is coordinated to the metal(II) ion leaving the other oxygen atoms available to react with the iron oxide surface. It is worth noting that (i) the distance between two oxygen atoms belonging to the two Pyipa ligands is equal to $2.59 \AA$ very close to the distance between two oxygen atoms 


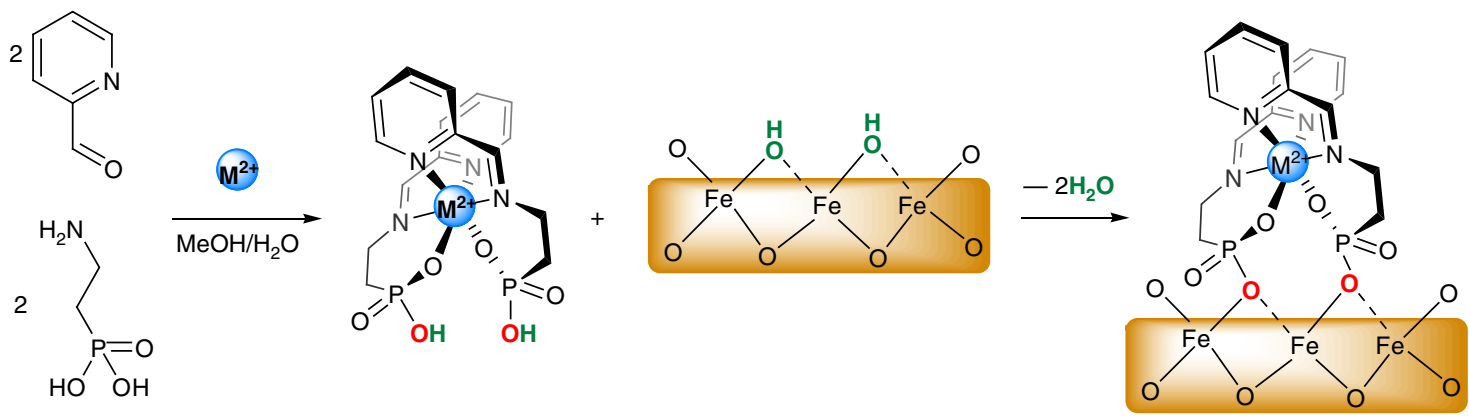

Figure 1 | Synthesis of the complexes and surface deposition. Schematic view of the formation of the metal ion containing complexes ( $\mathrm{M}=\mathrm{Co}$ and $\mathrm{Ni}$ ) and their anchoring to the metal iron-oxide substrate by the formation of M-OPO-Fe bonds and the releasing of water molecules.

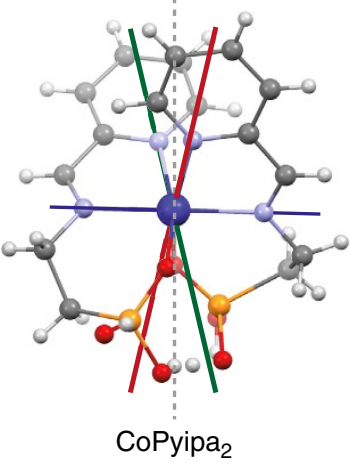

C

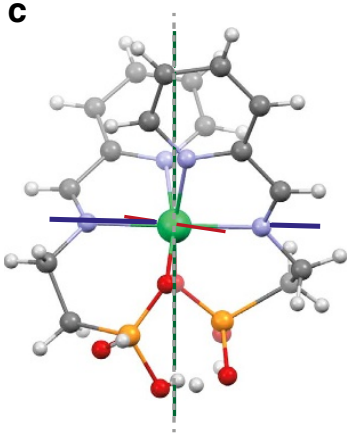

$\mathrm{NiPyipa}_{2}$ b

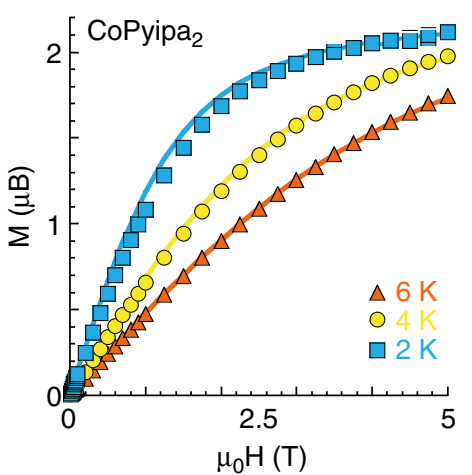

d

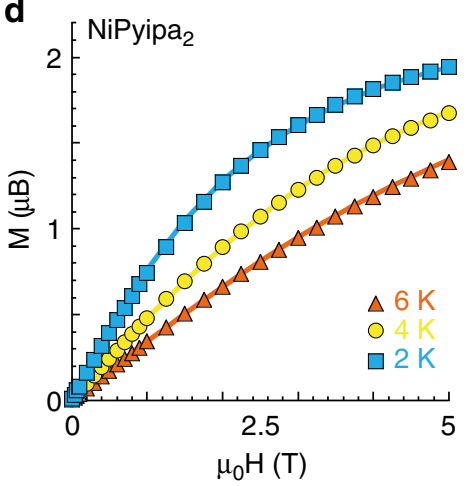

Figure 2 | Complex Co- and Ni(Pyipa) 2. X-ray crystal structure of $\mathrm{Co}(\text { Pyipa })_{2}$ (a) and $\mathrm{Ni}(\text { Pyipa })_{2}$ (c); $\mathrm{C}$, grey; $\mathrm{N}$, lilac; O, red; $\mathrm{P}$, orange; $\mathrm{H}$, white; $\mathrm{Co}$, blue; $\mathrm{Ni}$, green. The red, green and blue axes represent the $x, y$, $z$ direction of the anisotropy tensor, respectively. The magnetization as a function of field plots for $\mathrm{Co}(\text { Pyipa })_{2}$ (b) and $\mathrm{Ni}(\text { Pyipa })_{2}$ (d) solid lines correspond to the best fits; see $\mathrm{SI}$ for the parameter values.

belonging to the $\mathrm{Fe}_{3} \mathrm{O}_{4}$ (111) surface (2.85 $\AA$ ) ensuring little deformation of the coordination sphere of the metal ions upon the grafting of the complexes onto the substrate.

The magnetic behaviour of the complexes was studied by direct current (d.c.) magnetic susceptibility and magnetization measurements (Fig. 1; Supplementary Figs 1 and 2) and by $a b$ initio calculations that used the spin-orbit state interaction method (Supplementary Discussion: 'A $b$ initio calculations of the complexes' $)^{25-27}$. The calculations and the experimental data are in good agreement and indicate the presence of an easy plane of magnetization for $\mathrm{Co}(\text { Pyipa })_{2}$ and an easy axis for $\mathrm{Ni}(\text { Pyipa })_{2}$ (Table 1). We extracted the orientation of the magnetization axis from the $a b$ initio calculations, and we found that the hard and

\begin{tabular}{|c|c|c|}
\hline & Co(Pyipa) ${ }_{2}$ & $\mathrm{Ni}(\text { Pyipa })_{2}$ \\
\hline \multicolumn{3}{|l|}{ Experimental } \\
\hline$D\left(\mathrm{~cm}^{-1}\right)$ & +30.2 & -5.2 \\
\hline$E\left(\mathrm{~cm}^{-1}\right)$ & +5.0 & 0.0 \\
\hline \multicolumn{3}{|l|}{ Calculation } \\
\hline$D\left(\mathrm{~cm}^{-1}\right)$ & +32.2 & -3.4 \\
\hline$E\left(\mathrm{~cm}^{-1}\right)$ & +4.1 & +0.7 \\
\hline
\end{tabular}

easy axis of magnetization were perpendicular to the $\mathrm{C}_{2}$ symmetry axis for $\mathrm{Co}(\text { Pyipa })_{2}$, and $\mathrm{Ni}(\text { Pyipa })_{2}$, respectively.

Before grafting the molecules onto iron oxide, we checked their integrity by performing X-ray photoelectron spectroscopy (XPS) on crystalline samples dissolved in methanol and casted on a graphite substrate (HOPG). We measured the XPS of a fresh and of a 2-day incubated solution (as in the case of the grafting procedure on the magnetic substrate). The XPS spectra at the N1s and P2p edges are almost identical for the four samples (Supplementary Figs 3-6; Supplementary Table 2). The analysis of the spectra allowed quantification of the relative percentages of three elements ( $\mathrm{M}, \mathrm{N}$ and $\mathrm{P}$ ) for each sample, which is in agreement for the expected theoretical ratio 1:4:2 for M:N:P (Supplementary Table 2; Supplementary Discussion: 'XPS analysis').

Monolayer preparation and characterization. A monolayer of molecules (Co- or $\mathrm{Ni}(\text { Pyipa })_{2}$ ) on epitaxial $\mathrm{Fe}_{3} \mathrm{O}_{4}$ (111) was obtained by self-assembly from solution (see Methods for details). This reaction is well documented and has been widely used $^{17,20-24}$, and proceeds in the following manner: the hydroxyl groups present on the surface are protonated by the phosphonic acid of the complex leading to $\mathrm{PO}^{-}$species that react with the iron ions of the substrate causing the expulsion of water molecules (Fig. 1) ${ }^{17}$. We monitored surface coverage by atomic force microscopy (AFM) and time-of-flight secondary ion mass spectrometry (ToF-SIMS) (Fig. 3; Supplementary Figs 7-10). AFM data revealed homogenous coverage of the substrates (Fig. 3b; Supplementary Fig. 7) with the surface profile indicating a height of the objects that is consistent with the complexes being deposited onto the surface $(\sim 1 \mathrm{~nm})$. By comparing the AFM images before and after surface deposition (Fig. 3b; Supplementary Fig. 7a), we note the absence of degradation of the surface (we do not observe holes or aggregates), and we note an 
a

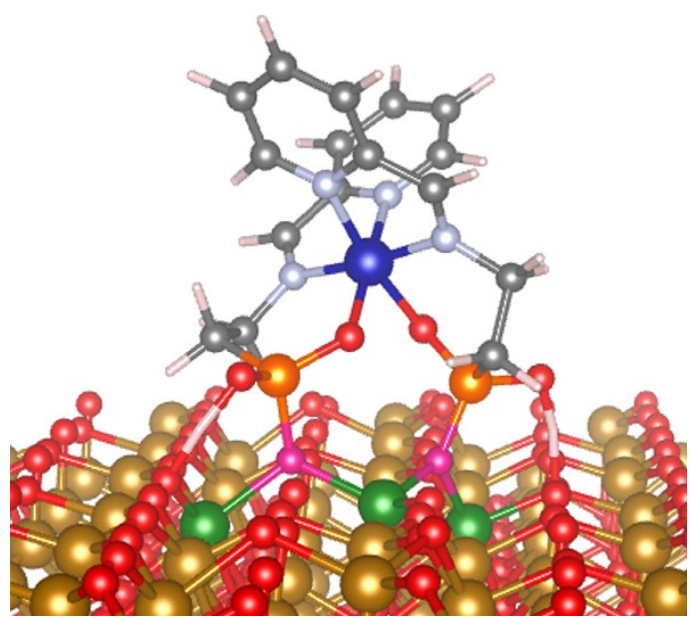

b

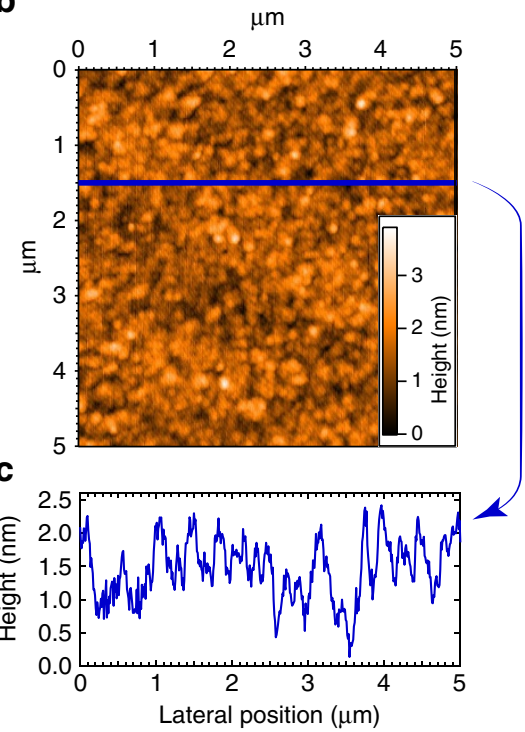

Figure 3 | Monolayer of $\mathbf{C o}\left(\mathbf{P y i p a}_{\mathbf{2}}\right.$ on epitaxial $\mathbf{F e}_{\mathbf{3}} \mathbf{O}_{\mathbf{4}}$. (a) Schematic representation of the DFT minimized orientation of $\mathrm{Co}(\mathrm{Pyipa})_{2}$ anchored onto epitaxial $\mathrm{Fe}_{3} \mathrm{O}_{4}$ : C, grey; $\mathrm{N}$, lilac; $\mathrm{O}$, red or magenta when bound to a phosphonate; $\mathrm{P}$, orange; $\mathrm{H}$, white; $\mathrm{Co}$, blue; $\mathrm{Ni}$, green; $\mathrm{Fe}$, gold or green when bound to the molecule; (b) AFM image of a monolayer of $\mathrm{Co}(\text { Pyipa })_{2}$ anchored onto epitaxial $\mathrm{Fe}_{3} \mathrm{O}_{4}$; (c) Surface profile of the monolayer of $\mathrm{Co}(\mathrm{Pyipa})_{2}$.

increase in the height of the surface profile, which is consistent with a monolayer on the surface. We, however, wanted to use a different and complementary technique to ensure the formation of chemical bonds between the complexes and the substrate. We thus performed Tof-SIMS studies that allowed detecting the molecular fragments confirming the presence of the molecules on the iron oxide substrates. Positive and negative ToF-SIMS spectra (Supplementary Figs 8-10; Supplementary Tables 2-3) showed a variety of fragments confirming that the phosphonic acid moiety binds to the iron oxide via the oxygen $\left(\mathrm{Fe}-\mathrm{O}-\mathrm{P}(\mathrm{OR})_{2}\right)$ as assumed. It also showed larger fragments (Co(Pyipa)-Fe, Ni(Pyipa)-Fe) indicating that the ligand is bound to both the iron of the surface and the metal ion of the complex. Particularly, the detection of fragments such as $\left[\mathrm{M}\left(\mathrm{C}_{5} \mathrm{H}_{4} \mathrm{NCHN}\right)_{2}\right]^{+},\left[\mathrm{M}\left(\mathrm{PO}_{3}\right)_{2} \mathrm{FeO}_{2}\right]^{-}$, and related ones (Supplementary Figs 8-10; Supplementary Tables 3-4) that contain parts of the two tridentate ligands linked to $M$ confirms that the intact complexes ( $\mathrm{M}$ and the two tridentate ligands) are chemically linked to the substrate. It is important to note that if the phosphonates were weakly bound to the iron oxide surface (that is, hydrogen bonded or mere physical adsorption), we would not expect complex fragments containing both iron and phosphonate to be present. Similar fragmentation was found in ToF-SIMS data of octadecylphosphoric acid on tantalum oxide surfaces ${ }^{22}$. The above-mentioned observations indicate that our molecules remain intact during the deposition process, and that they chemically bind to the surface via the phosphonate group. Of course, we could have some unreacted molecules present, but we believe that our thorough washing steps (coupled with absence of aggregates in the AFM images) are sufficient to remove all unreacted molecules from the surface. The XPS spectra of monolayers of complexes were too noisy to be quantitatively analysed. However, X-ray absorption (XAS) spectra of bulk (on graphite) and grafted monolayers are identical (see below); the presence of large amount of damaged molecules linked to the substrate will lead to different XAS spectra for the bulk and grafted samples. In addition, the observation of the angular dependence of the magnetic behaviour of the Co-grafted substrate (see below) excludes the presence of damaged molecular fragments that would be statistically oriented on the surface. In summary, the combination of the results from the three techniques (XPS, TOF-SIMS and XAS) ensures that the Co- and Ni-containing complexes keep their integrity when reacted with the $\mathrm{Fe}_{3} \mathrm{O}_{4}$ substrates and that are indeed chemically linked to the surface.

DFT calculations of the surface. Since the epitaxial $\mathrm{Fe}_{3} \mathrm{O}_{4}(111)$ surface has three different sites with $\mathrm{O}-\mathrm{O}$ distances close to that of the $\mathrm{OH}-\mathrm{OH}$ separation of the complexes (see structure description of the molecules above), the preferential coordination sites of the substrate together with the orientation of the molecules was determined by numerical simulations ${ }^{17}$. Determination of the equilibrium state and energy minimum of the system has been performed using density functional theory (DFT) molecular dynamics technique (FIREBALL) ${ }^{28-31}$. In our calculations, we set the molecule onto a surface of $\mathrm{Fe}_{3} \mathrm{O}_{4}$ oriented along the (111) direction formed by octahedral $\left(\mathrm{O}_{h}\right)$ and tetrahedral $\left(\mathrm{T}_{\mathrm{d}}\right) \mathrm{Fe}$ ions bridged by oxo ligands ${ }^{32}$. We initially positioned the molecule in three different configurations (a physisorbed and two chemisorbed ones, see SI for details). The orientation of the molecule was chosen such that the anchoring phosphonate moieties pointed towards the surface with the different possible binding modes ${ }^{17}$. Since the physisorbed energy is not compatible with the experimental rinse cycles (the molecular layer is stable after this operation), we decided to directly simulate the anchoring of the molecule by substituting the surface oxygen atoms by the phosphonates one (this configuration is otherwise out of range of standard DFT optimization due to a strong potential barrier between the physisorbed and an anchored state). We proceeded to do a DFT molecular dynamic simulation at room temperature to reproduce the experimental conditions of molecular deposition on the surface, followed by a structural optimization at $0 \mathrm{~K}$ to determine the final configuration on the surface. This procedure gave a stable structure and the molecular adsorption energies of the three configurations (Supplementary Discussion: 'Density Functional Theory-Geometrical optimization of the complexes on the surface' $)^{33}$. Further magnetic optimization was performed on 
these structures to obtain an accurate description of the magnetic state of each molecule. The most stable orientation corresponds to the condition where the $\mathrm{C}_{2}$ symmetry axis of the complexes is perpendicular to the surface as expected from the orientation of the oxygen phosphonate anchoring groups (Fig. 3a); the two oxygen atoms belonging to the two different phosphonates (phos) have replaced two oxygen atoms (bridging octahedral and tetrahedral $\mathrm{Fe}$ ions) belonging to the surface creating $\mathrm{Fe}-\mathrm{O}_{\text {phos }}$ coordination bonds. This configuration leads to the molecules being linked to three $\mathrm{Fe}$ atoms through $\mathrm{O}_{\text {phos }}\left(\mathrm{Fe}_{\text {oct }}-\mathrm{O}_{\text {phos }}-\mathrm{Fe}_{\text {tet }}{ }^{-}\right.$ $\mathrm{O}_{\text {phos }}-\mathrm{Fe}_{\text {oct }}$ ), as highlighted in Figs 1 and 3a. It is important to note that there are three possible anchoring sites for the complexes on the $\mathrm{Fe}_{3} \mathrm{O}_{4}$ (111) surface with $\mathrm{O}-\mathrm{O}$ distances of 2.85, 2.97 and 3.08 $\AA$. The most stable situation was found for the molecules bound on the site with O-O distance of $2.85 \AA$, which is reasonable because the distance between the two oxygen atoms of the phosphonic acid groups $\left(\mathrm{O}_{\text {phos }}\right)$ is $2.59 \AA$. In addition, the length of the molecule measured perpendicular to the $C_{2}$ axis ( $7.5 \AA$ ) excludes its grafting on all the binding sites and ensures an almost total coverage of the surface even if only one over six sites in average are occupied with a complex. For $\mathrm{Ni}(\text { Pyipa })_{2}$, the easy axis of magnetization found for the isolated complex thus is parallel to the surface, while the hard axis found for the isolated $\mathrm{Co}(\text { Pyipa })_{2}$ complex is parallel to the surface and thus the easy plane is perpendicular to the surface.

Magnetic characterization of the monolayers. To attain the sensitivity required to probe a monolayer of molecules, element specific magnetic properties were investigated by recording the magnetic dichroic component of the XAS of the surface $(\mathrm{Fe})$ and of the complexes (Co and $\mathrm{Ni}$ for $\mathrm{Co}-$ and $\mathrm{Ni}(\mathrm{Pyipa})_{2}$, respectively) at the $\mathrm{L}_{2,3}$ edges. Circular polarized X-rays were employed for two incidence angles $\left(\theta=0^{\circ}\right.$ and $\left.45^{\circ}\right)$ between the sample normal and the X-ray propagation vector. All measurements were recorded at $2 \mathrm{~K}$ in the total yield electron mode with the magnetic field aligned parallel to the photon propagation vector ${ }^{7,34}$. The XAS spectra, and the resulting XMCD spectra $\left(\sigma^{-}-\sigma^{+}\right)$, at the iron, cobalt and nickel $\mathrm{L}_{2,3}$ edges were acquired in the presence of a 6.5 $\mathrm{T}$ field employing the two circular polarizations $\left(\sigma^{+} ; \sigma^{-}\right)$. The XAS and XMCD spectra at the $\mathrm{Fe}_{2,3}$ edges of the epitaxial $\mathrm{Fe}_{3} \mathrm{O}_{4}$ surface before and after molecule grafting are similar (Supplementary Figs 11 and 12), and are comparable to those published in the literature ${ }^{35}$, demonstrating that the surface deposition method did not damage the substrates. The XAS and XMCD spectra at the Ni and the Co edges show a strong dichroic signal that is indicative of molecules deposited on the surface (Fig. 4; Supplementary Fig. 13). Note that the non-flat background for the XMCD for Co comes from the XMCD signal of the $\mathrm{Fe} \mathrm{L}_{2,3}$ edges and can be removed by recording the XMCD on a pure $\mathrm{Fe}_{3} \mathrm{O}_{4}$ surface in the energy range of the $\mathrm{Co}_{2,3}$ edges as shown in Supplementary Fig. 14. The signals were compared with those recorded on a thick film of the same complexes deposited by drop casting (Supplementary Fig. 15). The spectra are similar, and confirmed that the electronic structure of the molecule is retained upon grafting. The shape of the XMCD signal at the $\mathrm{Fe}_{2} \mathrm{~L}_{3}$ edge (Supplementary Fig. 12) corresponds to the situation where the magnetic moments of the octahedral $\mathrm{Fe}$ sites are aligned with the magnetic field (negative XMCD signal) and are antiparallel to those of the tetrahedral sites (positive XMCD signal), which correspond to an antiferromagnetic coupling between octahedral and tetrahedral sites $^{35}$. The XMCD signal at the $\mathrm{Co}_{3}$ edge is aligned with the magnetic field (negative XMCD), therefore, it is aligned with the octahedral $\mathrm{Fe}$ sites. The identical scenario is true for $\mathrm{Ni}$.

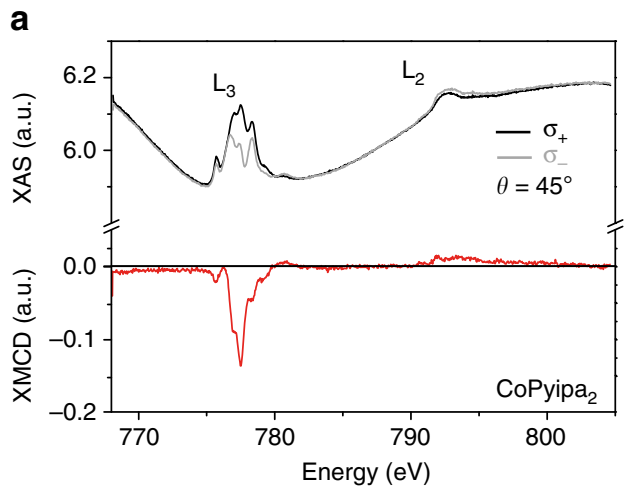

C

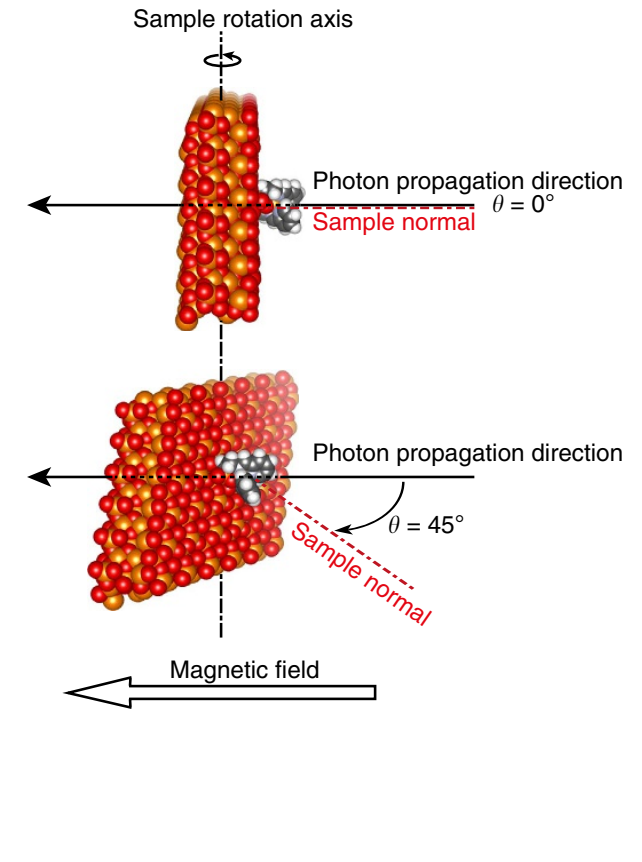

Figure 4 | XAS/XMCD spectra of a monolayer of Co- and Ni(Pyipa) 2. (a) Cobalt, and (b) Nickel $L_{2,3}$ edges XAS (black and grey line) and XMCD (red lines) spectra recorded at $T=2 \mathrm{~K}$, and $\theta=45^{\circ}$ using left $\left(\sigma_{+}\right)$and right hand $\left(\sigma_{-}\right)$circularly polarized light in $6.5 \mathrm{~T}$ field; (c) schematic representation of the measurement geometry. 
A sum rule analysis ${ }^{36,37}$ was performed to extrapolate the values of the spin and orbital magnetic moments for $\mathrm{Co}$ and $\mathrm{Ni}$ in the complex deposited as a monolayer on the $\mathrm{Fe}_{3} \mathrm{O}_{4}$ surface at $\theta=0^{\circ}$ and $45^{\circ}$, and as a thick film at $\theta=45^{\circ}$. This analysis allows to obtain the spin and orbital magnetic moments from the integrated XMCD signals, and gave the results summarized in Table 2 (see Supplementary Information for the details of the calculations). Interestingly the Co magnetic moment found corresponds to one third of the saturation magnetization of $3 \mu_{\mathrm{B}}$ for an isotropic $S=3 / 2$ cobalt ion. This result confirms that the ground state of the $\mathrm{Co}^{\mathrm{II}}$ ion is mainly built from $M_{S}= \pm 1 / 2$

\begin{tabular}{|c|c|c|c|}
\hline Sample & $\mathbf{M}_{\mathbf{L}}=-\boldsymbol{\mu}_{\mathbf{B}}<\mathbf{L}_{\mathbf{Z}}>$ & $\boldsymbol{M}_{\mathbf{S}}=-\boldsymbol{g}_{\mathrm{o}} \boldsymbol{\mu}_{\mathbf{B}}<\mathbf{S}_{\mathbf{Z}}>$ & $M_{L} / M_{S}$ \\
\hline $\begin{array}{l}\text { Co monolayer } \\
\theta=0^{\circ}\end{array}$ & $0.39 \pm 0.03 \mu_{\mathrm{B}}$ & $1.19 \pm 0.08 \mu_{\mathrm{B}}$ & $0.33 \pm 0.11 \mu_{\mathrm{B}}$ \\
\hline $\begin{array}{l}\text { Co monolayer } \\
\theta=45^{\circ}\end{array}$ & $0.44 \pm 0.03 \mu_{\mathrm{B}}$ & $1.39 \pm 0.08 \mu_{\mathrm{B}}$ & $0.28 \pm 0.11 \mu_{\mathrm{B}}$ \\
\hline $\begin{array}{l}\text { Ni monolayer } \\
\theta=0^{\circ}\end{array}$ & $0.35 \pm 0.02 \mu_{\mathrm{B}}$ & $1.39 \pm 0.12 \mu_{\mathrm{B}}$ & $0.25 \pm 0.14 \mu_{\mathrm{B}}$ \\
\hline $\begin{array}{l}\text { Ni monolayer } \\
\theta=45^{\circ}\end{array}$ & $0.34 \pm 0.02 \mu_{\mathrm{B}}$ & $1.50 \pm 0.12 \mu_{\mathrm{B}}$ & $0.23 \pm 0.14 \mu_{\mathrm{B}}$ \\
\hline $\begin{array}{l}\text { Co thick film } \\
\theta=45^{\circ}\end{array}$ & $0.44 \pm 0.03 \mu_{\mathrm{B}}$ & $1.25 \pm 0.08 \mu_{\mathrm{B}}$ & $0.36 \pm 0.11 \mu_{\mathrm{B}}$ \\
\hline $\begin{array}{l}\text { Ni thick film } \\
\theta=45^{\circ}\end{array}$ & $0.17 \pm 0.02 \mu_{\mathrm{B}}$ & $1.34 \pm 0.12 \mu_{\mathrm{B}}$ & $0.13 \pm 0.14 \mu_{\mathrm{B}}$ \\
\hline
\end{tabular}

Table 2 | Values of the spin and orbital magnetic moments or $\mathrm{Co}_{0}$ and $\mathrm{Ni}$ in the complex deposited as a monolayer on the $\mathrm{Fe}_{3} \mathrm{O}_{4}$ surface at $\theta=0^{\circ}$ and $45^{\circ}$, and as a thick film at $\theta=\mathbf{4 5}^{\circ}$.

a

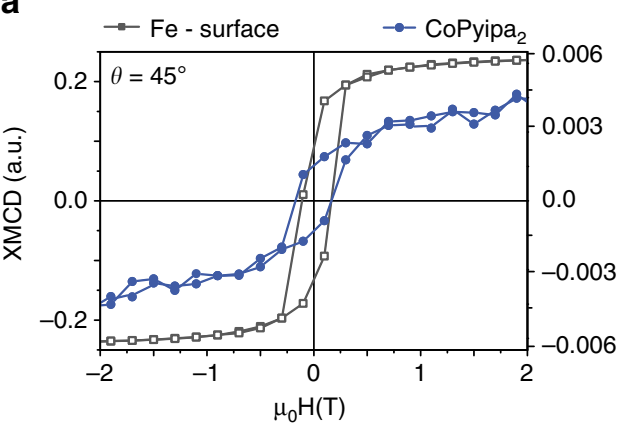

C

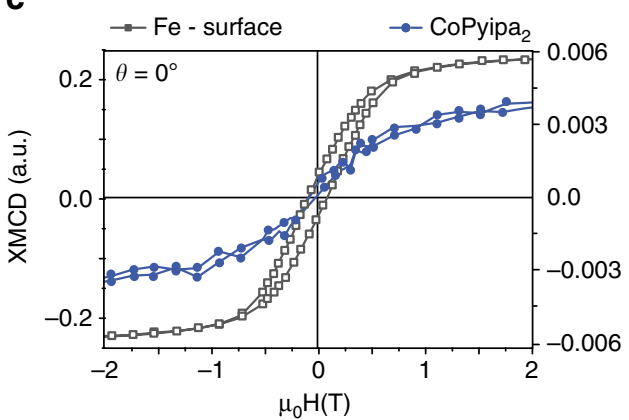

with very small contributions from higher $M_{S}( \pm 3 / 2)$ terms, exactly what was found from the magnetization measurements analysis (Supplementary Discussion: 'Sum rule calculations'). It is important to note that X-ray sum rule analysis assumes a transition between two well-defined shells (that is, $2 p$ to $3 d$ valence state transition for transition metal ions), and that the $3 d$ valence states are separable from the other state. Also, from the literature $^{38}$ we do not expect a large deviation of the values of $\left\langle T_{\mathrm{z}}\right\rangle$ (magnetic dipole term) as the complexes are hexacoordinated so no real structural anisotropy is present. Thus, we neglected this term in our calculations.

To gain insight on the nature of the interaction of $\mathrm{Co}$ - and $\mathrm{Ni}$ (Pyipa $)_{2}$ with the epitaxial $\mathrm{Fe}_{3} \mathrm{O}_{4}$ surface, we measured element-specific XMCD-detected hysteresis loops. The fielddependence $\mathrm{Fe}$, $\mathrm{Co}$ and $\mathrm{Ni} \mathrm{L}_{3} \mathrm{XMCD}$ intensity (multiplied by -1$)$ at fixed photon energy and normalized are shown in Fig. 5 and Supplementary Fig. 16 for the geometry where the $\mathrm{X}$-ray propagation vector is at $45^{\circ}$ and $0^{\circ}$ with the sample normal i.e. the substrate makes an angle of $45^{\circ}$ and $0^{\circ}$ with the applied magnetic field (Fig. 3c). It was possible to observe an opening of the magnetic hysteresis loop for $\mathrm{Co}$ (Pyipa) $)_{2}$ (Fig. 5a), while no opening occurs when the magnetic field is perpendicular to the substrate (Fig. 5c; Supplementary Fig. 16a). These results indicate that the Co-containing complex is magnetically coupled to the $\mathrm{Fe}_{3} \mathrm{O}_{4}$ (111) surface and that the molecules are magnetically oriented otherwise no angular dependence of the magnetization would be observed. To investigate the magnetic behaviour of $\mathrm{Co}$ (Pyipa $)_{2}$ isolated from the $\mathrm{Fe}_{3} \mathrm{O}_{4}$ (111) surface, an amorphous non-magnetic ultrathin insulating layer $\left(1 \mathrm{~nm}\right.$ of $\left.\mathrm{Al}_{2} \mathrm{O}_{3}\right)$ was deposited between the iron oxide surface and the molecules. It is important to note that the same type of reactivity is possible on $\mathrm{Al}_{2} \mathrm{O}_{3}$ layer as for $\mathrm{Fe}_{3} \mathrm{O}_{4}$ and other oxides but no structural

b

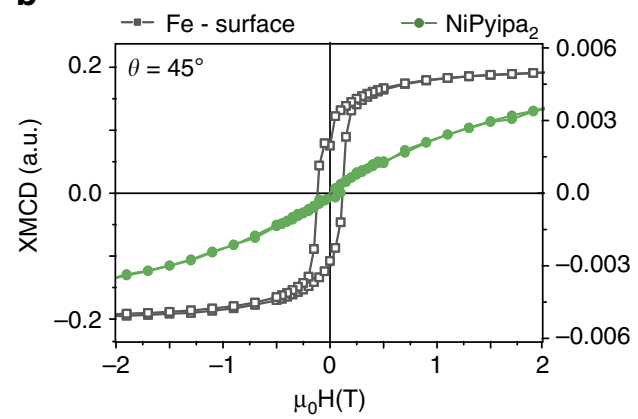

d

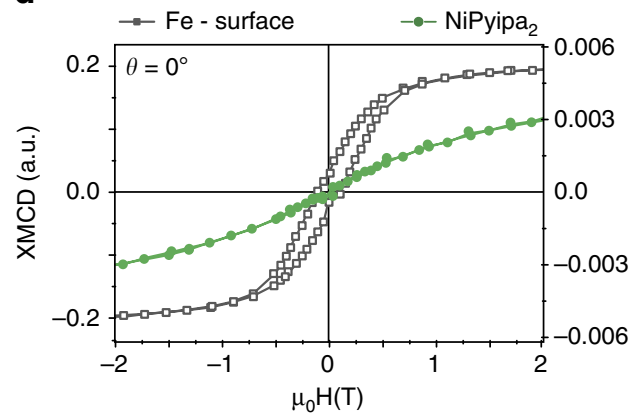

Figure 5 | Element-specific field dependence of the magnetization of the complexes and surface (Fe). Hysteresis curves (multiplied by -1 ) of the Co atoms (blue), Ni atoms (green) and Fe atoms (grey) obtained at the $\mathrm{L}_{2,3}$ edges XMCD maxima at $T=2 \mathrm{~K}$, and $\theta=45^{\circ}$. (Monochromatized $\mathrm{X}$-rays are set at the energy of the maximum absolute value of the XMCD signal (that is, $h v=777.5 \mathrm{eV}$ for $\mathrm{Co}$, $h v=851 \mathrm{eV}$ for Ni, and $h v=707 \mathrm{eV}$ for Fe) then the external magnetic field is switched step by step from $+6.5 \mathrm{~T}$ down to $-6.5 \mathrm{~T}$ and back to $+6.5 \mathrm{~T}$. At each step the magnetic field is switched from left to right circular polarization to yield the element specific magnetization curves. 
a

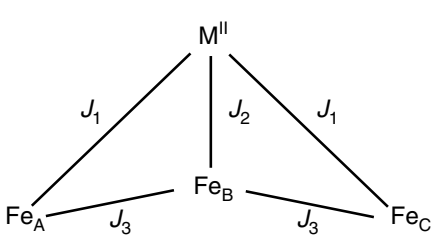

C

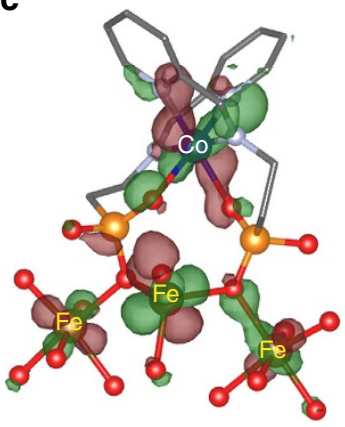

MO220 b
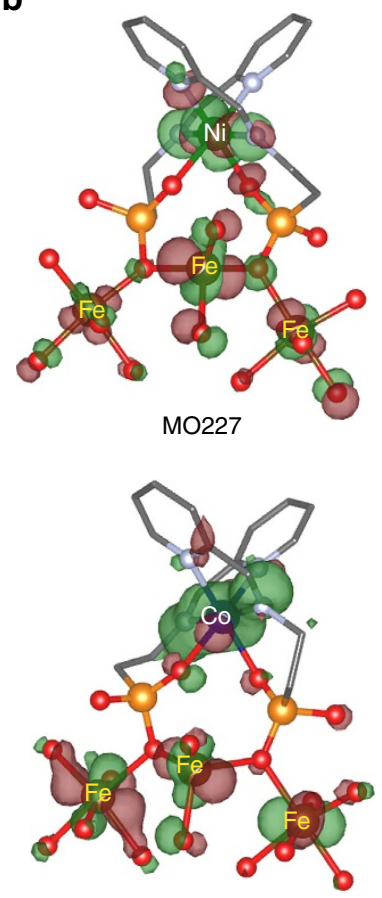

MO224
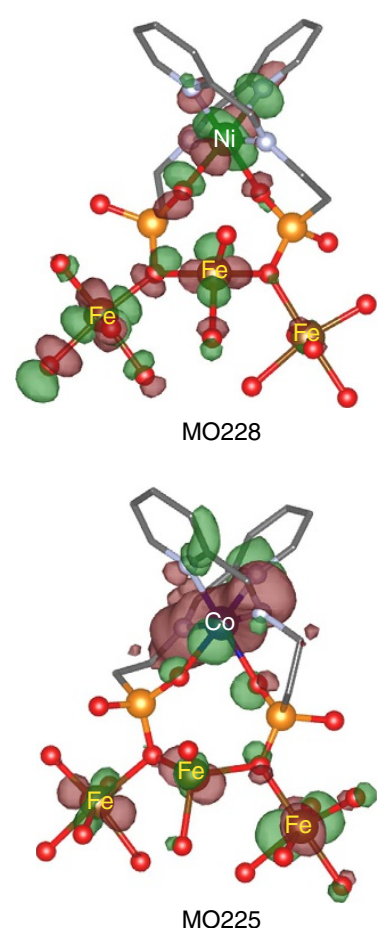

Figure 6 | Geometry of the exchange interaction. (a) Interaction topology between the $\mathrm{MPyipa}_{2}(\mathrm{M}=\mathrm{Ni}$ or $\mathrm{Co})$ and the surface Fe ions. Optimized geometry of the singly occupied magnetic orbitals for $\mathrm{Ni}(\text { Pyipa })_{2}$ (b) and $\mathrm{Co}(\text { Pyipa })_{2}$ (c). Positive (red) and negative (green) isosurfaces for the high-spin solution. $\mathrm{C}$, grey; $\mathrm{N}$, lilac; $\mathrm{O}$, red; $\mathrm{P}$, orange; $\mathrm{Co}$, blue; $\mathrm{Ni}$, green; Fe, dark orange; hydrogen atoms were omitted for clarity. The $\mathrm{MO}$ labels are in ascending energy levels.

orientation of the molecules is expected because the $\mathrm{Al}_{2} \mathrm{O}_{3}$ layer is amorphous ${ }^{17,20-24}$. In this system, the XAS and XMCD spectra are similar to those when the molecules are grafted on iron oxide (Supplementary Fig. 17), but no opening of the magnetization loop was seen for $\theta=45^{\circ}$ (Supplementary Fig. 18). These data indicate that the hysteresis loop for the $\mathrm{Co} / \mathrm{Fe}_{3} \mathrm{O}_{4}$ system is due to the magnetic coupling between the molecules and the iron-oxide because in the presence of the diamagnetic $\mathrm{Al}_{2} \mathrm{O}_{3}$ where no magnetic coupling is expected no hysteresis loop is observed.

Furthermore, since the loop is open at $45^{\circ}$, the easy plane of magnetization of the anchored complex is parallel to the substrate or the anisotropy nature of the molecule has changed upon coupling to have an easy axis of magnetization that is parallel to substrate. The experimental data cannot rule out between these two possibilities: if the anchored complexes had their easy axes of magnetization parallel to the surface, the magnetic behaviour would be the same as if each molecule had an easy plane parallel to the substrate because the azimuthal distribution of the easy axes. This last hypothesis implies that the nature of the magnetic anisotropy of the complex has changed upon coupling with the substrate from an easy plane to an easy axis (parallel to the substrate). While the first hypothesis implies that the complex kept its anisotropy nature (easy plane of magnetization that contains its $\mathrm{C}_{2}$ symmetry axis when isolated, Fig. 3a) but switched its orientation to be perpendicular to the $C_{2}$ axis (thus parallel to the substrate) upon coupling with the magnetic substrate. This interpretation is supported by the values of the magnetic moment extracted from the sum rule applied on Co for the two orientations (Table 2) where a slight increase is observed for the $45^{\circ}$. A precise determination of the anisotropy tensor axes and to rule out one of the two hypotheses require further studies at several orientation of the magnetic field. No opening of the magnetization loop was seen for $\mathrm{Ni}(\text { Pyipa })_{2}$ (Fig. 5b,d;
Supplementary Fig. 16b) for the two orientations highlighting the absence of (or the presence of very weak) magnetic coupling with the substrate. We reasoned that the difference in coupling between $\mathrm{Ni}$ (Pyipa) ${ }_{2}$ (absence of hysteresis) and $\mathrm{Co}$ (Pyipa) (presence of hysteresis) excludes dipole-dipole interactions between $\mathrm{Co}$ (Pyipa $)_{2}$ and substrate, as such interactions would have resulted in the same behaviour for the two molecules. The magnetic coupling between $\mathrm{Co}$ (Pyipa) $)_{2}$ and $\mathrm{Fe}_{3} \mathrm{O}_{4}$ thus is due to exchange.

Broken symmetry calculations. To elucidate the nature and relative magnitude of this exchange coupling, and the differences between the magnetic behaviour of Co- and $\mathrm{Ni}(\text { Pyipa })_{2}$ when anchored to the surface, we carried out broken symmetry calculations using the B3LYP functional of the GAUSSIAN 09 package (Supplementary Discussion: 'Density Functional Theory - calculation of the magnetic coupling between the molecules the surface' $)^{39-43}$. We employed the extended basis sets: Valence Triple Zeta plus Polarization (VTZP) for Fe, Ni, Co and for the ligand atoms ${ }^{44-46}$. Starting from the results of the DFT molecular dynamic simulation described above, we optimized the geometries of the complexes and their anchoring to the surface. The system is composed of the three coupled $\mathrm{Fe}^{\mathrm{III}}$ ions $(S=5 / 2)$ $\left(\mathrm{Fe}^{\mathrm{III}}{ }_{\mathrm{Oh}}-\mathrm{Fe}^{\mathrm{III}}{ }_{\mathrm{Td}}-\mathrm{Fe}^{\mathrm{III}} \mathrm{Oh}\right)$ as explained above, we assume that the surface $\mathrm{Fe}^{\mathrm{II}}$ atoms were oxidized to $\left.\mathrm{Fe}^{\mathrm{III}}\right)^{47}$ surrounded by their coordinated oxygen atoms (from the surface) and the complexes either $\mathrm{Co}(\text { Pyipa })_{2}\left(\mathrm{Co}{ }^{\mathrm{II}}, S=3 / 2\right)$ or $\mathrm{Ni}(\text { Pyipa })_{2}\left(\mathrm{Ni}^{\mathrm{II}}, S=1\right)$. The Heisenberg Hamiltonian $\hat{H}=J_{1}\left(\hat{S}_{\mathrm{A}} \cdot \hat{S}_{\mathrm{M}}+\hat{S}_{\mathrm{C}} \hat{S}_{\mathrm{M}}\right)+J_{2}\left(\hat{S}_{\mathrm{B}} \cdot \hat{S}_{\mathrm{M}}\right)+$ $J_{3}\left(\hat{S}_{\mathrm{A}} \cdot \hat{S}_{\mathrm{B}}+\hat{S}_{\mathrm{B}} \cdot \hat{S}_{\mathrm{C}}\right)$ describes the low energy spectrum of the four spin centres: $\mathrm{M}=\mathrm{Ni}^{\mathrm{II}}$ or $\mathrm{Co}{ }^{\mathrm{II}}, \hat{S}_{B}=$ the spin operator of the central tetracoordinated $\mathrm{Fe}_{\mathrm{A}}^{\mathrm{III}}$ ion, $\hat{S}_{A}$ and $\hat{S}_{C}=$ the spin operators of its hexacoordinated neighbors $\mathrm{Fe}_{\mathrm{A}}^{\mathrm{III}}$ and $\mathrm{Fe}_{\mathrm{C}}^{\mathrm{III}}$, respectively 
(Fig. 6a). The analytical energies of the four possible computed solutions are given in Supplementary Fig. 19. The Hamiltonian was chosen so that when the exchange parameters $\left(J_{\mathrm{i}}\right)$ are positive the coupling is antiferromagnetic.

First, we calculated the surface coupling in the absence of Co- or $\mathrm{Ni}(\text { Pyipa })_{2}\left(J_{1}=J_{2}=0\right)$. As expected for an epitaxial $\mathrm{Fe}_{3} \mathrm{O}_{4}$ (111) surface, we found that the surface $\mathrm{Fe}^{\mathrm{III}}$ have an antiferromagnetic coupling $\left(J_{3}=52 \mathrm{~cm}^{-1}\right)$. The ferromagnetic solution was $1,321 \mathrm{~cm}^{-1}$ higher in energy than the antiferromagnetic one. Second, we computed the energies in the presence of Co- or $\mathrm{Ni}(\text { Pyipa })_{2}$.

By computing the energies of the different solutions based on the coupling scheme depicted in Fig. 6a, we find that the coupling between the $\mathrm{Ni}^{\mathrm{II}}$ (or $\mathrm{Co}^{\mathrm{II}}$ ) ions and the surface is independent from the nature (ferromagnetic or antiferromagnetic) of the exchange coupling within the substrate (Supplementary Discussion: 'Density Functional Theory-calculation of the magnetic coupling between the molecules the surface'). From the energy differences between the various solutions (Supplementary Table 5) we obtained $J_{1}=18 \mathrm{~cm}^{-1}, J_{2}=-$ $2 \mathrm{~cm}^{-1}, J_{3}=-40 \mathrm{~cm}^{-1}$ for $\mathrm{Ni}(\text { Pyipa })_{2}$, and $J_{1}=-54 \mathrm{~cm}^{-1}$, $J_{2}=-49 \mathrm{~cm}^{-1} ; J_{3}=-47 \mathrm{~cm}^{-1}$ for Co(Pyipa $)_{2}$. This translates into a weakly antiferromagnetic exchange coupling between the $\mathrm{Ni}$ (Pyipa $)_{2}$ and the surface and a strongly ferromagnetic coupling for $\mathrm{Co}(\text { Pyipa })_{2}$. It is worth noting two important points. (1) The values of $J$ are not exact values, but rather their magnitude should be considered. (2) The calculations suggest that the exchange between tetrahedral and octahedral Fe ions became ferromagnetic upon grafting. It is unfortunately difficult to confirm this theoretical result from the present experimental data because, due to the size of the molecule, only a weak percentage of the $\mathrm{Fe}$ ions are linked to the complexes; dedicated specific measurements comparing bare and grafted samples are needed to reach an unambiguous conclusion.

The opposite nature of the exchange interaction can be explained by the difference in the molecular orbitals (MO) depicted in Fig. 6. None of the singly occupied molecular orbitals (SOMOs) of $\mathrm{Co}$ (Pyipa) $)_{2}$ have a strong overlap with the $\mathrm{Fe}^{\mathrm{III}}$ SOMOs through the bridging oxygen ligands (see MOs number 220, 224 and 225 in Fig. 6). The most important physical factor contributing to the exchange coupling of $\mathrm{Co}(\mathrm{Pyipa})_{2}$ with the surface is brought by the direct exchange integrals between all the magnetic SOMOs, which are always ferromagnetic ${ }^{48,49}$, and the spin polarization. On the contrary, one of the two SOMOs of $\mathrm{Ni}(\text { Pyipa })_{2}$ (MO number 228 (Fig. 6)) exhibits an extended delocalization tail on the oxygen atom bridging the complex to the surface through the phosphonate anchoring group. The overlap between this $\mathrm{Ni}$ (Pyipa) $)_{2}$ SOMO and the $\mathrm{Fe}^{\mathrm{III}}$ one brings a large kinetic contribution (antiferromagnetic) to the exchange coupling that compensates and overcomes the ferromagnetic contribution and leads to an overall antiferromagnetic interaction, though weaker in magnitude than the ferromagnetic one in the case of Co (refs 48,49). As the magnetic orbitals of the Ni(II) ion are lower in energy than those of the $\mathrm{Co}$ (II) ion, the interaction between the occupied orbitals of the ligand and those of the metal ions are stronger for the $\mathrm{Ni}$ (II) complex than for the $\mathrm{Co}(\mathrm{II})$ one. For this reason, the $\mathrm{Ni}$ (II) ion magnetic orbitals (of $\sigma$ nature) are more delocalized over the ligands than those of the $\mathrm{Co}(\mathrm{II})$ ion. As these interactions mediate the coupling between the complexes and the surface, a stronger antiferromagnetic contribution to the magnetic coupling is expected for the $\mathrm{Ni}$ (II) complex, thus compensating the ferromagnetic contribution and leading ultimately to an overall weaker magnetic coupling than for $\mathrm{Co}(\mathrm{II})$. The difference between the nature and the magnitude of the couplings is also corroborated by the shorter $\mathrm{M}^{\mathrm{II}}$ spacer/surface distance for $\mathrm{Ni}(\text { Pyipa })_{2}$ than for $\mathrm{Co}(\text { Pyipa })_{2}$, strengthening the antiferromagnetic superexchange mechanism. It is important to note that the absolute numerical values of $J_{\mathrm{i}}$ have to be considered with caution due to the approximations made in the calculations. The much stronger ferromagnetic coupling of the $\mathrm{Co}^{\mathrm{II}}$ centre with the surface compared with the $\mathrm{Ni}^{\mathrm{II}}$ centre explains the opening (or lack thereof) of the magnetic hysteresis for $\mathrm{Co}(\text { Pyipa })_{2}$ (or $\left.\mathrm{Ni}(\text { Pyipa })_{2}\right)_{\text {) when deposited }}$ onto the ferrimagnetic surface. XMCD measurements at lower temperatures (XMCD below $1 \mathrm{~K}$ is not accessible on the DEIMOS line) would likely provide further indications on the nature of the magnetic coupling in the $\mathrm{Ni}(\text { Pyipa })_{2}$ system $^{7,8}$.

\section{Discussion}

The experimentally observed change in the anisotropy tensor of the Co-containing molecules is mainly due to exchange coupling with the ferromagnetic substrate and not to a change of the local structure of the molecules that might have occurred upon grafting. The following three points support this conclusion: (i) the local structure of the grafted molecules, optimized by DFT, shows no important changes in the metal-ligand bond distances and angles. (ii) The zero-field splitting value of the isolated molecules is relatively large $\left(+30 \mathrm{~cm}^{-1}\right)$, and only a large change of the local Co environment is necessary to alter the single ion anisotropy tensor. (iii) The magnitude of the exchange coupling between the Co-containing complex and the substrate (computed by DFT) is of the same order of magnitude as the anisotropy energy of the isolated complex; an alteration of the anisotropy tensor of the isolate molecules is thus expected due to exchange coupling as experimentally observed. If the exchange coupling were much weaker than the anisotropy energy of the isolated molecules, no change in the single ion anisotropy tensor of the isolated molecules would have been detected.

In conclusion, we have shown that by molecular design, we can chemically anchor metal-containing molecules to a magnetic iron oxide electrode and we can finely control the molecule/magnetic surface interface structure. The coupling between the magnetic molecules and $\mathrm{Fe}_{3} \mathrm{O}_{4}$ is due to exchange and not to dipole-dipole interactions. The chemical anchoring responsible of the exchange magnetic coupling switches the orientation of the easy plane of magnetization of the $\mathrm{Co}$ (II) complex or changes the nature of its magnetic anisotropy from an easy plane to an easy axis of magnetization. The electronic structure of the metal ion has a paramount importance on the nature of the coupling: $\mathrm{Co}$ (Pyipa $)_{2}$ undergoes a strong ferromagnetic coupling with the substrate while the structurally analogous $\mathrm{Ni}(\mathrm{Pyipa})_{2}$ is either not coupled or weakly antiferromagnetically coupled to the substrate. We can, therefore, envision that controlling the molecule/substrate exchange interaction would allow tuning the spin current (sign of the magneto-resistance) at the interface and eventually lead to using such current to switch the magnetization of single anisotropic molecules in molecule-based spintronic devices.

\section{Methods}

General. Epitaxial $\mathrm{Fe}_{3} \mathrm{O}_{4}(111)$ thin films were grown by molecular beam epitaxy on $\alpha-\mathrm{Al}_{2} \mathrm{O}_{3}$ (0001) according to literature procedures ${ }^{50}$. Unless otherwise stated, all reagents were purchased from Aldrich or TCI and used without further purification. Electrospray ionization mass spectrometry (ESI-MS) spectra were recorded on a Thermo Scientific 2009 mass spectrometer. IR spectra were recorded on a Bruker TENSOR-27 Fourier transform infrared (FT-IR) spectrometer equipped with an attenuated total reflectance (ATR crystal diamond/ZnSe) sample holder in the $4000-500 \mathrm{~cm}^{-1}$ range. Elemental analysis was taken on a Thermo Scientific Flash analyzer.

Synthesis of Co(Pyipa) 2. (Cobaltl(II) bis-2-[(Pyridin-2-ylmetylene)amino]ethyl phosphonic acid). A round bottom flask was charged with 2-aminoethylphosphonic acid $(77.8 \mathrm{mg}, 0.622 \mathrm{mmol}), 2$-pyridinecarboxaldehyde $(58 \mu \mathrm{l}$, $0.610 \mathrm{mmol}), \mathrm{Co}\left(\mathrm{CH}_{3} \mathrm{COO}\right)_{2} \cdot 4 \mathrm{H}_{2} \mathrm{O}(75 \mathrm{mg}, 0.301 \mathrm{mmol}), \mathrm{MeOH}(4 \mathrm{ml})$ and demineralized $\mathrm{H}_{2} \mathrm{O}(4 \mathrm{ml})$. The reaction mixture was heated at reflux for $3 \mathrm{~h}$. The 
solvent was evaporated and a red product was obtained. The product was purified by vapor diffusion of $\mathrm{Et}_{2} \mathrm{O}$ into a $\mathrm{MeOH}$ solution of $\mathrm{Co}$ (Pyipa) $)_{2}(m=109.5 \mathrm{mg}$; yield $=74 \%)$. IR $\left(\mathrm{v} / \mathrm{cm}^{-1}\right): 3,317(\mathrm{br}), 2,946(\mathrm{br}), 2,888(\mathrm{br}), 2,529(\mathrm{br}), 2,162(\mathrm{~s})$, $1,981(\mathrm{br}), 1,780(\mathrm{~m}), 1,660(\mathrm{~s}), 1,626(\mathrm{~m}), 1,589(\mathrm{~m}), 1,571(\mathrm{~m}), 1,469(\mathrm{br}), 1,446$ (s), 1,366 (br), 1,352 (br), 1,286 (s), 1,226 (s), 1,156 (s), 1,104 (m), 1,091 (m), 1,051 (m), 1,033 (m), 1,010 (s), $988(\mathrm{~s}), 962(\mathrm{~m}), 937(\mathrm{~s}), 897(\mathrm{~s}), 875(\mathrm{~s}), 813(\mathrm{~m}), 785$ (m), $744(\mathrm{~m}), 707$ (br), $662(\mathrm{~s}), 634(\mathrm{~m})$. ESI-MS: $\mathrm{m} / z 486.03\left(\left[\mathrm{Co}(\text { Pyipa })_{2}\right]^{+}\right)$. Elem anal. Calcd for $\mathrm{C}_{16} \mathrm{H}_{24} \mathrm{~N}_{4} \mathrm{O}_{8} \mathrm{P}_{2}$ Co: C, 36.87; H, 4.64; N, 10.75. Found: C, $36.80 ; \mathrm{H}, 4.82 ; \mathrm{N}, 10.73$.

Synthesis of Ni(Pyipa) 2. (Nickel(II) bis-2-[(Pyridin-2-ylmetylene)amino]ethyl phosphonic acid). A round bottom flask was charged with 2-aminoethylphosphonic acid $(85.0 \mathrm{mg}, 0.680 \mathrm{mmol}), 2$-pyridinecarboxaldehyde $(64 \mu \mathrm{l}$, $0.673 \mathrm{mmol}), \mathrm{Ni}\left(\mathrm{CH}_{3} \mathrm{COO}\right)_{2} \cdot 4 \mathrm{H}_{2} \mathrm{O}(80 \mathrm{mg}, 0.321 \mathrm{mmol}), \mathrm{MeOH}(4 \mathrm{ml})$ and demineralized $\mathrm{H}_{2} \mathrm{O}(4 \mathrm{ml})$. The reaction mixture was heated at reflux for $3 \mathrm{~h}$. The solvent was evaporated and a red product was obtained. The product was purified by vapor diffusion of $\mathrm{Et}_{2} \mathrm{O}$ into a $\mathrm{MeOH}$ solution of $\mathrm{Ni}(\text { Pyipa })_{2}(m=98.6 \mathrm{mg}$; yield $=67 \%)$. IR $\left(\mathrm{v} / \mathrm{cm}^{-1}\right): 3,317(\mathrm{br}), 2,946(\mathrm{br}), 2,888(\mathrm{br}), 2,529(\mathrm{br}), 2,162(\mathrm{~s})$, 1,981 (br), 1,780 (m), 1,660 (s), 1,626 (m), 1,589 (m), 1,571 (m), 1,469 (br), 1,446 (s), 1,366 (br), 1,352 (br), 1,286 (s), 1,226 (s), 1,156 (s), 1,104 (m), 1,091 (m), 1,051 (m), 1,033 (m), 1,010 (s), $988(\mathrm{~s}), 962(\mathrm{~m}), 937(\mathrm{~s}), 897(\mathrm{~s}), 875(\mathrm{~s}), 813(\mathrm{~m}), 785$ $(\mathrm{m}), 744(\mathrm{~m}), 707(\mathrm{br}), 662(\mathrm{~s}), 634(\mathrm{~m})$. ESI-MS: $m / z 485.04\left(\left[\mathrm{Ni}(\mathrm{Pyipa})_{2}\right]^{+}\right)$. Elem anal. Calcd for $\mathrm{C}_{16} \mathrm{H}_{28} \mathrm{~N}_{4} \mathrm{O}_{10} \mathrm{P}_{2} \mathrm{Ni}$ : C, 34.50; $\mathrm{H}, 5.07 ; \mathrm{N}, 10.06$. Found: $\mathrm{C}$, $34.37 ; \mathrm{H}, 4.54 ; \mathrm{N}, 10.02$.

Single crystal X-ray diffraction studies. X-ray diffraction data were collected by using a Kappa X8 APPEX II Bruker diffractometer with graphite-monochromated $\mathrm{Mo}_{\mathrm{K} \alpha}$ radiation $(\lambda=0.71073 \AA$ ). Crystals were mounted on a CryoLoop (Hampton Research) with Paratone-N (Hampton Research) as cryoprotectant and then flashfrozen in a nitrogen-gas stream at $100 \mathrm{~K}$. The temperature of the crystal was maintained at the selected value $(100 \mathrm{~K})$ by means of a 700 series Cryostream cooling device to within an accuracy of $\pm 1 \mathrm{~K}$. The data were corrected for Lorentz polarization, and absorption effects. The structures were solved by direct methods using SHELXS-97 (ref. 51) and refined against $F^{2}$ by full-matrix least-squares techniques using SHELXL-97 (ref. 52) with anisotropic displacement parameters for all non-hydrogen atoms. Hydrogen atoms were located on a difference Fourier map and introduced into the calculations as a riding model with isotropic thermal parameters. All calculations were performed by using the Crystal Structure crystallographic software package WINGX ${ }^{53}$.

Molecular magnetic measurements. The magnetic susceptibility measurements were obtained using a Quantum Design SQUID magnetometer MPMS-XL7 operating between 1.8 and $300 \mathrm{~K}$ for d.c.-applied fields ranging from -5 to $5 \mathrm{~T}$. Dc analysis was performed on polycrystalline samples of $\mathrm{Co}$ - and $\mathrm{Ni}(\mathrm{Pyipa})_{2}(17.61 \mathrm{mg}$ and $17.31 \mathrm{mg}$, respectively) wrapped in eicosan under a field between 0.1 and $1 \mathrm{~T}$ and between 1.8 and $300 \mathrm{~K}$. The $\chi_{M} T=f(\mathrm{~T})$ curve for $\mathrm{Ni}(\mathrm{Pyipa})_{2}$ shows a Curie-law behaviour between 300 and $50 \mathrm{~K}$ (ground state with no first order orbital momentum ${ }^{3} \mathrm{~A}_{2 \mathrm{~g}}$ ) and then decreases indicating the presence of a zero-field splitting (ZFS) within the $S=1$ state (Supplementary Fig. 1). While, for Co(Pyipa) a steady decrease is observed from room temperature down to $75 \mathrm{~K}$ and then more rapidly, in line with a ground state with a non completely quenched orbital momentum as expected for slightly distorted octahedral for $\mathrm{Co}^{\mathrm{II}}$ complexes ${ }^{54}$. The data for $\mathrm{Co}-$ and $\mathrm{Ni}(\mathrm{Pyipa})_{2}$ were fitted by a full diagonalization of the energy matrices considering many orientations of the magnetic field. The best fits lead to the following parameters: $D_{\mathrm{Ni}}=-5.2 \mathrm{~cm}^{-1}, E_{\mathrm{Ni}}=0, g_{\mathrm{Ni}}=2.22$, $R$ (agreement factor) $=2 \times 10^{-5}, D_{\mathrm{Co}}=+30.2 \mathrm{~cm}^{-1}, E_{\mathrm{Co}}=5.0 \mathrm{~cm}^{-1}, g_{\mathrm{Co}}=2.3$, $R=5 \times 10^{-4}$. $D$ and $E$ are the axial and the rhombic ZFS parameters, and $g$ is the Lande factor of the spin Hamiltonian $\hat{H}=g \mu_{\mathrm{B}} \mathbf{H} \bullet \mathbf{S}+D\left[\hat{S}_{z}^{2}-S(S+1) / 3\right]+E$ $\left(\hat{S}_{x}^{2}-\hat{S}_{y}^{2}\right)$. These parameters are related to the $D$ tensor matrix elements by $2 E=\left|D_{x x}-D_{y y}\right|$ and $D=3 D_{z z} / 3$.

Monolayer preparation. The monolayers were prepared by immersion of the $\mathrm{Fe}_{3} \mathrm{O}_{4}$ substrates into $10 \mathrm{ml}$ of a freshly filtered $0.5 \mathrm{mM}$ solution of either $\mathrm{Co}(\text { Pyipa })_{2}$ or $\mathrm{Ni}(\text { Pyipa })_{2}$ dissolved in $\mathrm{MeOH}$. After 3 days of immersion the substrates were removed from the solution and rinsed thoroughly with neat solvent. All the monolayer preparation experiments were carried out at room temperature

\section{Geometrical optimization of the geometry of the molecule on the surface.} The FIREBALL package uses a localized optimized minimal basis set ${ }^{29}$ and the self-consistency is achieved over the occupation numbers through the Harris functional ${ }^{30}$. The LDA exchange-correlation energy is calculated using the efficient multi centre weighted exchange correlation density approximation (McWEDA). ${ }^{31}$

X-ray photoelectron spectroscopy. was carried out using monochromatized $\mathrm{Al} \mathrm{K}$ alpha1 X-rays $(h v=1,486.6 \mathrm{eV})$, a hemispherical analyzer, and a channel plate detector. The spectrometer was calibrated at the Au $4 \mathrm{f}$ core level at a binding energy of $84 \mathrm{eV}$. Spectra were recorded at a takeoff angle of $90^{\circ}$. The pass energy was set to $160 \mathrm{eV}$ for survey and $20 \mathrm{eV}$ for core level, giving an energy resolution of $0.38 \mathrm{eV}$.

Atomic force microscopy. AFM images were recorded using a Pico-LE microscope (Molecular Imaging-Agilent Technologies) in contact mode. AFM tips were Si-coated with $\mathrm{Pt} / \mathrm{Ir}$ alloy with a stiffness in between $0.1 \mathrm{~N} \mathrm{~m}^{-1}$ and $0.3 \mathrm{~N} \mathrm{~m}^{-1}$. The tip radius was given at $20 \mathrm{~nm}$.

Time-of-flight secondary ion mass spectrometry. Time-of-flight secondary ion mass spectrometry data were acquired using a TOF.SIMS V spectrometer (ION-TOF GmbH, Muenster, Germany). The analysis chamber was maintained at less than $5 \times 10^{-7} \mathrm{~Pa}$ under operational conditions. The total primary ion flux was less than $10^{12}$ ions $\mathrm{cm}^{-2}$ ensuring static conditions. A pulsed $25 \mathrm{keV} \mathrm{Bi}^{+}$primary ion source (Liquid Metal Ion Gun, LMIG) at a current of about $1 \mathrm{pA}$ (high current bunched mode), rastered over a scan area of $500 \times 500 \mu \mathrm{m}$ was used as the analysis beam. Data acquisition and processing analyses were performed using the commercial IonSpec program. The exact mass values of at least seven known species, from $\mathrm{H}^{-}, \mathrm{C}^{-}, \mathrm{C}^{-}{ }_{2}, \mathrm{C}^{-}{ }_{3}, \mathrm{PO}_{2}^{-}, \mathrm{PO}_{3}^{-}, \mathrm{FeO}^{-}, \mathrm{FeO}_{2}^{-}, \mathrm{Fe}_{2} \mathrm{O}^{-}, \mathrm{FeOPO}_{3}^{-}$, and $\mathrm{H}^{+}, \mathrm{CH}_{3}^{+}, \mathrm{Na}^{+}, \mathrm{Ca}^{+}, \mathrm{Fe}_{2}^{+}, \mathrm{C}_{5} \mathrm{H}_{5} \mathrm{Fe}^{+}, \mathrm{C}_{6} \mathrm{H}_{6} \mathrm{Fe}^{+}$, were used for calibration of the data, acquired in the negative and positive ion mode, respectively.

XAS/XMCD studies. The XAS/XMCD studies at the Fe, Co and $\mathrm{Ni}_{2,3}$ edges were carried out at the DEIMOS beam line, SOLEIL Synchrotron (Gif-sur-Yvette, France $)^{55,56}$. To ensure optimal detection sensitivity, the absorption spectra were measured in the Total Electron Yield mode. We used low density photons to avoid radiation damages to the samples. XMCD spectra were obtained from circularly polarized absorption spectra at $2 \mathrm{~K}$ under an applied magnetic field of $6.5 \mathrm{~T}$ parallel to the X-ray propagation vector. The XMCD-detected hysteresis loops were obtained at $2 \mathrm{~K}$ with the magnetic field sweeping ( -6.5 to $6.5 \mathrm{~T}$ ) parallel to the $\mathrm{X}$-ray propagation vector.

Data availability. The X-ray crystallographic coordinates for structures reported in this Article have been deposited at the Cambridge Crystallographic Data Centre (CCDC), under deposition numbers CCDC-1049643 and CCDC-1049644. These data can be obtained free of charge from The Cambridge Crystallographic Data Centre via www.ccdc.cam.ac.uk/data_request/cif. The remaining data that support the findings of this study are available from the corresponding authors upon reasonable request.

\section{References}

1. Bogani, L. \& Wernsdorfer, W. Molecular spintronics using single-molecule magnets. Nat. Mater. 7, 179-186 (2008).

2. Sanvito, S. Molecular spintronics. Chem. Soc. Rev. 40, 3336 (2011).

3. Barraud, C. et al. Unravelling the role of the interface for spin injection into organic semiconductors. Nat. Phys. 6, 615-620 (2010).

4. Blouzon, C., Ott, F., Tortech, L., Fichou, D. \& Moussy, J. B. Anti-ferromagnetic coupling in hybrid magnetic tunnel junctions mediated by monomolecular layers of $\alpha$-sexithiophene. Appl. Phys. Lett. 103, 042417 (2013).

5. Wende, H. et al. Substrate-induced magnetic ordering and switching of iron porphyrin molecules. Nat. Mater. 6, 516-520 (2007).

6. Schmaus, S. et al. Giant magnetoresistance through a single molecule. Nat. Nanotechnol. 6, 185-189 (2011).

7. Mannini, M. et al. Magnetic memory of a single-molecule quantum magnet wired to a gold surface. Nat. Mater. 8, 194-197 (2009).

8. Mannini, M. et al. Quantum tunnelling of the magnetization in a monolayer of oriented single-molecule magnets. Nature 468, 417-421 (2010).

9. Malavolti, L. et al. Magnetism of $\mathrm{TbPc}_{2} \mathrm{SMMs}$ on ferromagnetic electrodes used in organic spintronics. Chem. Commun. 49, 11506 (2013).

10. Lodi Rizzini, A. et al. Exchange biasing single molecule magnets: coupling of $\mathrm{TbPc}_{2}$ to antiferromagnetic layers. Nano Lett. 12, 5703-5707 (2012).

11. Scheybal, A. et al. Induced magnetic ordering in a molecular monolayer. Chem. Phys. Lett. 411, 214-220 (2005).

12. Annese, E., Casolari, F., Fujii, J. \& Rossi, G. Interface magnetic coupling of Fe-phthalocyanine layers on a ferromagnetic surface. Phys. Rev. B 87, 054420 (2013).

13. Auwärter, W., Écija, D., Klappenberger, F. \& Barth, J. V. Porphyrins at interfaces. Nat. Chem. 7, 105-120 (2015).

14. Rizzini, A. L. et al. Coupling of single, double, and triple-decker metalphthalocyanine complexes to ferromagnetic and antiferromagnetic substrates. Surf. Sci. 630, 361-374 (2014).

15. Klar, D. et al. Oxygen-tuned magnetic coupling of Fe-phthalocyanine molecules to ferromagnetic Co films. Phys. Rev. B 88, 224424 (2013).

16. Heinrich, B. W., Braun, L., Pascual, J. I. \& Franke, K. J. Tuning the magnetic anisotropy of single molecules. Nano Lett. 15, 4024-4028 (2015).

17. Mutin, P. H., Guerrero, G. \& Vioux, A. Hybrid materials from organophosphorus coupling molecules. J. Mater. Chem. 15, 3761 (2005). 
18. Gooding, J. J. \& Ciampi, S. The molecular level modification of surfaces: from self-assembled monolayers to complex molecular assemblies. Chem. Soc. Rev. 40, 2704 (2011).

19. Bauer, T. et al. Phosphonate- and carboxylate-based self-assembled monolayers for organic devices: a theoretical study of surface binding on aluminum oxide with experimental support. ACS Appl. Mater. Interfaces 5, 6073-6080 (2013).

20. White, M. A., Johnson, J. A., Koberstein, J. T. \& Turro, N. J. Toward the syntheses of universal ligands for metal oxide surfaces: controlling surface functionality through click chemistry. J. Am. Chem. Soc. 128, 11356-11357 (2006).

21. Tatay, S. et al. Self-assembled monolayer-functionalized half-metallic manganite for molecular spintronics. ACS Nano 6, 8753-8757 (2012).

22. Textor, M. et al. Structural chemistry of self-assembled monolayers of octadecylphosphoric acid on tantalum oxide surfaces. Langmuir 16, 3257-3271 (2000).

23. Queffélec, C., Petit, M., Janvier, P., Knight, D. A. \& Bujoli, B. Surface modification using phosphonic acids and esters. Chem. Rev. 112, 3777-3807 (2012).

24. Gagnon, K. J., Perry, H. P. \& Clearfield, A. Conventional and unconventional metal-organic frameworks based on phosphonate ligands: MOFs and UMOFs. Chem. Rev. 112, 1034-1054 (2012).

25. Roos, B. O. \& Malmqvist, P.-A. Relativistic quantum chemistry: the multiconfigurational approach. Phys. Chem. Chem. Phys. 6, 2919-2927 (2004).

26. Karlstroem, G. et al. MOLCAS: a program package for computational chemistry. Comput. Mater. Sci. 28, 222-239 (2003).

27. Malmqvist, P. A., Roos, B. O. \& Schimmelpfennig, B. The restricted active space (RAS) state interaction approach with spin-orbit coupling. Chem. Phys. Lett. 357, 230-240 (2002).

28. Lewis, J. P. et al. Advances and applications in the FIREBALL ab initio tightbinding molecular-dynamics formalism. Phys. Status Solidi B 248, 1989-2007 (2011).

29. Basanta, M. A., Dappe, Y. J., Jelínek, P. \& Ortega, J. Optimized atomic-like orbitals for first-principles tight-binding molecular dynamics. Comput. Mater. Sci. 39, 759-766 (2007).

30. Harris, J. Simplified method for calculating the energy of weakly interacting fragments. Phys. Rev. B 31, 1770-1779 (1985).

31. Jelínek, P., Wang, H., Lewis, J., Sankey, O. \& Ortega, J. Multicenter approach to the exchange-correlation interactions in $a b$ initio tight-binding methods. Phys. Rev. $B$ 71, 235101 (2005).

32. Pentcheva, R. et al. Jahn-Teller stabilization of a 'polar' metal oxide surface: $\mathrm{Fe}_{3} \mathrm{O}_{4}$ (001). Phys. Rev. Lett. 94, 126101 (2005).

33. Schull, G., Dappe, Y. J., González, C. s., Bulou, H. \& Berndt, R. Charge injection through single and double carbon bonds. Nano Lett. 11, 3142-3146 (2011).

34. Nakajima, R., Stohr, J. \& Idzerda, Y. U. Electron-yield saturation effects in L-edge X-ray magnetic circular dichroism spectra of Fe, Co, and Ni. Phys. Rev. B 59, 6421 (1999).

35. Carvallo, C. et al. Biogenic vs. abiogenic magnetite nanoparticles: a XMCD study. Am. Mineral. 93, 880-885 (2008).

36. Carra, P., Thole, B. T., Altarelli, M. \& Wang, X. X-ray circular dichroism and local magnetic fields. Phys. Rev. Lett. 70, 694-697 (1993).

37. Thole, B. T., Carra, P., Sette, F. \& van der Laan, G. X-ray circular dichroism as a probe of orbital magnetization. Phys. Rev. Lett. 68, 1943-1946 (1992).

38. Piamonteze, C., Miedema, P. S. \& de Groot, F. M. F. The accuracy of the spin sum rule in XMCD. J. Phys.: Conf. Ser. 190, 012015 (2009).

39. Frisch, M. J. et al. Gaussian 09 (Revision C.01) (Gaussian, Inc., Wallingford CT, 2009).

40. Bencini, A. \& Totti, F. DFT description of the magnetic structure of polynuclear transition-metal clusters: The complexes $\left[\left\{\mathrm{Cu}(\mathrm{bpca})_{2}\right.\right.$ $\left.\left.\left(\mathrm{H}_{2} \mathrm{O}\right)_{2}\right\}\left\{\mathrm{Cu}\left(\mathrm{NO}_{3}\right)_{2}\right\}_{2}\right],($ bpca $=$ Bis (2-pyridylcarbonyl) amine), and $\left[\mathrm{Cu}(\mathrm{DBSQ})\left(\mathrm{C}_{2} \mathrm{H}_{5} \mathrm{O}\right)\right]$ 2,(DBSQ $=3$, 5-di-tert-butyl-semiquinonato). Int. J. Quantum Chem. 101, 819-825 (2005).

41. Bencini, A. \& Totti, F. A few comments on the application of density functional theory to the calculation of the magnetic structure of oligo-nuclear transition metal clusters. J. Chem. Theory Comput. 5, 144-154 (2008).

42. Noodleman, L. \& Norman, Jr J. G. The X $\alpha$ valence bond theory of weak electronic coupling. application to the low-lying states of $\mathrm{Mo} 2 \mathrm{Cl} 84-$. J. Chem. Phys. 70, 4903-4906 (1979).

43. Noodleman, L. Valence bond description of antiferromagnetic coupling in transition metal dimers. J. Chem. Phys. 74, 5737-5743 (1981).

44. Wachters, A. J. H. Gaussian basis set for molecular wavefunctions containing third-row atoms. J. Chem. Phys. 52, 1033 (1970).

45. Hay, P. J. Gaussian basis sets for molecular calculations. The representation of $3 d$ orbitals in transition-metal atoms. J. Chem. Phys. 66, 4377 (1977).
46. Raghavachari, K. \& Trucks, G. W. Highly correlated systems. Excitation energies of first row transition metals Sc-Cu. J. Chem. Phys. 91, 1062 (1989).

47. Kaya, S., Ogasawara, H. \& Nilsson, A. Determination of the surface electronic structure of $\mathrm{Fe}_{3} \mathrm{O}_{4}\left(\begin{array}{lll}1 & 1 & 1\end{array}\right)$ by soft X-ray spectroscopy. Catal. Today 240, 184-189 (2015).

48. Malrieu, J. P. Cancellations occurring in the calculation of transition energies by a perturbation development of configuration interaction matrices. J. Chem. Phys. 47, 4555 (1967).

49. Anderson, P. W. New approach to the theory of superexchange interactions. Phys. Rev. Lett. 115, 2-13 (1959).

50. Ramos, A. V. et al. Magnetotransport properties of $\mathrm{Fe}_{3} \mathrm{O}_{4}$ epitaxial thin films: thickness effects driven by antiphase boundaries. J. Appl. Phys. 100, 103902 (2006).

51. SHELXS-97. Program for Crystal Structure Solution (University of Göttingen, 1997).

52. SHELXL-97. Program for the Refinement of Crystal Structures from Diffraction Data (University of Göttingen, 1997).

53. Farrugia, L. J. WinGX suite for small-molecule single-crystal crystallography. J. Appl. Crystallogr 837-838 (1999).

54. Kahn, O. Molecular Magnetism (VCH, 1993).

55. Henke, B. L., Liesegang, J. \& Smith, S. D. Soft-X-ray-induced secondaryelectron emission from semiconductors and insulators: Models and measurements. Phys. Rev. B 19, 3004-3021 (1979).

56. Ohresser, P. et al. DEIMOS: a beamline dedicated to dichroism measurements in the 350-2500 eV energy range. Rev. Sci. Instrum. 85, 013106 (2014).

\section{Acknowledgements}

This work is supported by a public grant overseen by the French National Research Agency (ANR) as part of the 'Investissements d'Avenir' program 'Labex NanoSaclay' (reference: ANR-10-LABX-0035). We acknowledge SOLEIL for provision of synchrotron radiation facilities. This work was performed using HPC resources from CALMIP (Grant 2015-1517).

\section{Author contributions}

V.E.C., M.T., J.-B.M. and T.M. planned and conceived the experiments and study. M.T. and I.C. synthesized and characterized the molecules. J.-B.M. provided the surfaces. R.G. collected and analysed the single crystal X-ray data. M.T., E.R., V.E.C. and T.M. executed the magnetic characterization. L.T., M.T. and I.C. took the AFM images. F.M. recorded the XPS spectra and analysed them with L.T. and J.-B.M. Y.J.D. performed the DFT molecular simulations. V.E.C., M.T., T.M., E.O., P.O, P.S., M.-A.A., R.M., P.S. and S.D collected and analysed the XMCD data. N.G., V.G.C., F.K and N.S performed the $a b$ initio calculations on the isolated molecules and the molecules on the surface. A.G., V.E.C. and T.M. collected and analysed the Tof-SMIs data. All authors participated in the discussion. V.E.C. and T.M. wrote the paper with contributions from all authors.

\section{Additional information}

Supplementary Information accompanies this paper at http://www.nature.com/ naturecommunications

Competing financial interests: The authors declare no competing financial interests.

Reprints and permission information is available online at http://npg.nature.com/ reprintsandpermissions/

How to cite this article: Campbell, V.E. et al. Engineering the magnetic coupling and anisotropy at the molecule-magnetic surface interface in molecular spintronic devices. Nat. Commun. 7, 13646 doi: 10.1038/ncomms13646 (2016).

Publisher's note: Springer Nature remains neutral with regard to jurisdictional claims in published maps and institutional affiliations.

This work is licensed under a Creative Commons Attribution 4.0 International License. The images or other third party material in this article are included in the article's Creative Commons license, unless indicated otherwise in the credit line; if the material is not included under the Creative Commons license, users will need to obtain permission from the license holder to reproduce the material. To view a copy of this license, visit http://creativecommons.org/licenses/by/4.0/

\footnotetext{
(C) The Author(s) 2016
} 